\title{
PROPERTIES OF EXCITABLE SITES IN THE SQUID AXON MEMBRANE AS REVEALED BY USE OF CHEMICAL STIMULANTS AND A SPECTRUM ANALYZER
}

\author{
Ichiji TASAKI \\ Laboratory of Neurobiology, National Institutes of Mental Health, \\ Bethesda, Maryland, U. S. A. \\ and \\ Marine Biological Laboratory, Woods Hole, \\ Massachusetts, U.S. A.
}

\begin{abstract}
Properties of the excitable sites in the squid axon membrane were studied by using various chemical stimulants and a real-time spectrum analyzer. Intact squid axons immersed in media with a reduced divalent cation concentration develop very small electric responses (1$30 \mu \mathrm{V}$ in amplitude) which repeat at more-or-less regular intervals. The frequency of repetition of these miniature responses falls when the temperature of the axon is lowered. Replacement of a small fraction of the external Na-ion with $\mathrm{K}$-ion is a powerful means of generating miniature responses. Passage of an outwardly directed current through the axonal membrane also evokes miniature responses. This effect of an electric current is attributed to a transport of intracellular $\mathrm{K}$-ion into the axonal membrane. The results of the effects of external application of the salts of $\mathrm{Li}$-, $\mathrm{Rb}$ - and $\mathrm{Cs}$-ion indicate that the ability of alkali metal ions to induce miniature response falls in the following order:
\end{abstract}

$\mathrm{K}, \mathrm{Rb}>\mathrm{Cs}>\mathrm{Na}>\mathrm{Li}$.

Peripheral nerve fibers can be excited, as is well known, by a variety of chemical stimulants. The simplest and most extensively studied form of chemical stimulant is lowering of the divalent cation concentration in the surrounding medium (see ARVANITAKI, 1939; BRINK et al., 1946). Initiation of action potentials by a chemical stimulant is always preceded by the appearance of oscillatory subthreshold responses. The amplitude of these oscillatory responses is known to vary with time in an apparently irregular manner, although the frequency of oscillation is more-or-less fixed under given experimental conditions (ARVANITAKI, 1939;

Received for publication August 8, 1977

田崎一二 
INOUE et al., 1973). Action potentials are released when the membrane potential reaches the critical level at the peaks of these oscillatory responses.

Quite recently, it was noted that a commercially available spectrum analyzer can be used to study physiological properties of extremely small oscillatory responses induced by chemical stimulants (Matsumoto et al.; Tasaki, in preparation). With this device, it is possible to determine Fourier components of apparently irregular electric signals on line. By applying this method to the squid axon membrane, the existence of extremely small oscillatory responses (1-30 $\mu \mathrm{V}$ peak-to-peak across the membrane) was demonstrated. The power spectrum of these "oscillatory miniature responses" was found to be approximately Lorentzian (Matsumoto et al., in preparation). Furthermore, these responses were found to be sensitive to both tetrodotoxin and tetraethylammonium ions (Tasaki, in preparation).

This paper describes the results of further studies of oscillatory miniature responses in squid giant axons conducted by the use of a real-time spectrum analyzer. The effects of temperature changes and of addition of the salts of alkali metals to the external medium on the frequency and amplitude of the miniature responses are described. The influence of passing electric currents through the axonal membrane on these responses is also described. Several interesting physicochemical properties of the macromolecules occupying discrete excitable sites in the axonal membrane are revealed by these studies.

\section{METHODS}

Giant nerve fibers (400-450 $\mu \mathrm{m}$ in diameter) of the squid, Loligo pealii, available in Marine Biological Laboratory, Woods Hole, Massachusetts, U.S.A., were used exclusively. Most of the small nerve fibers attached to the giant fiber were removed under a dissecting microscope. A giant fiber was mounted horizontally across a $30 \mathrm{~mm}$ wide Lucite chamber provided with two pairs of platinum electrodes, one pair located near each end of the giant fiber. The electrode used for recording variations in the intracellular potential was made of a fine $(25 \mu \mathrm{m}$ in diameter) platinum wire enclosed in a glass tubing of about $80 \mu \mathrm{m}$ in outside diameter. Only the platinized tip (about $50 \mu \mathrm{m}$ in length) of the electrode was exposed to make contact with the axoplasm. (The use of a glass-pipette electrode filled with an isotonic $\mathrm{KCl}$ solution for recording yielded substantially the same results (Tasa$\mathrm{ki}$, in preparation.) The saline solution in which the fiber was immersed was grounded with a large coil of platinum wire (see Fig. 1, top). After establishing that the fiber in the chamber was capable of developing normal action potentials (about $110 \mathrm{mV}$ in amplitude), all the extracellular electrodes (except the ground) were usually disconnected.

The intracellular recording electrode was led to a Bak unity-gain electrometer (Life Science). The output of the electrometer stage was connected to a Tektronix 
type 122 low-level preamplifier with a constant amplification of approximately $19 d_{b}$ in the range of frequency between 0.2 and $10,000 \mathrm{~Hz}$. The output of the preamplifier was led to a Model 440 A of the Mini-Ubiquitous Spectrum Analyzer of Nicolet Scientific Corp. A Tektronix 7704 oscilloscope was used to photograph the frequency spectra with a Polaroid camera. In order to improve the signal-to-noise ratio, most of the spectra presented in this paper were taken after averaging the signals 32 times; the time required for this procedure of averaging was approximately $13 \mathrm{sec}$. To study the effects of electric currents (see Fig. 4), an averaging time of about $3 \mathrm{sec}$ was adopted, because both the amplitude and the peak-frequency of the responses were continuously changing with time. The recording device was frequently calibrated with attenuated sinusoidal voltage waves.

The composition of the artificial sea water used contained the following concentrations (expressed in $\mathrm{mM}$ ) of salts: $423 \mathrm{NaCl}, 9 \mathrm{KCl}, 9.3 \mathrm{CaCl}_{2}, 23 \mathrm{MgCl}_{2}$, $25.5 \mathrm{MgSO}_{4}$, and a small amount of tris-(hydroxymethyl)-aminomethane buffer (pH 8.0-8.1). The following isotonic solutions of various salts were also used: $340 \mathrm{CaCl}_{2}, 530 \mathrm{NaCl}, 530 \mathrm{KCl}$, etc.; the $\mathrm{pH}$ of these solutions was adjusted to 8.0 8.1 with a small amount of tris- $\mathrm{HCl}$ buffer. The room temperature was between 19 and $20^{\circ} \mathrm{C}$.

\section{RESULTS}

\section{The amplitude and peak-frequency of oscillatory miniature responses}

In freshly excised squid giant axons immersed in normal sea water, extremely small signals with a frequency of about $150 \mathrm{~Hz}$ were often observed with a recording electrode inserted into the interior of the axon. When such a small signals was photographed, the intracellular electrode was withdrawn from the axon interior and another spectral record was taken with the recording tip of the electrode immersed in the sea water outside the axon. An example of such superposed photographs is reproduced in Fig. 1 (see Record A). Since the frequency of the signals arising from these axons was always very close to that of much larger responses observed in media with a reduced divalent cation concentration, it was inferred that these small signals represent physiologically significant responses of the giant axon.

When the divalent cation concentration of the external medium was reduced by a factor of 3 to 6 by mixing normal sea water with an isotonic $\mathrm{NaCl}$ solution, there was always a gradual increase in the amplitude of the signal at about $150 \mathrm{~Hz}$. (This procedure brought about no detectable fall of the membrane potential.) During the course of 10 to $30 \mathrm{~min}$ after application of a salt solution with a reduced divalent cation concentration, the amplitude of the signal was found to increase from an almost undetectable level to about $30 \mu \mathrm{V}$, peak-to-peak. During this period, both the peak-frequency and the bandwidth of the spectrum remained almost unaltered. However, the irregularity of the spectrum, which was frequently 
seen when the signal amplitude was very small (see Record C in Fig. 1), was found to decrease with increasing signal amplitude. This irregularity can easily be understood if one assumes that these signals are developed by a small number of excitable sites in the axonal membrane.
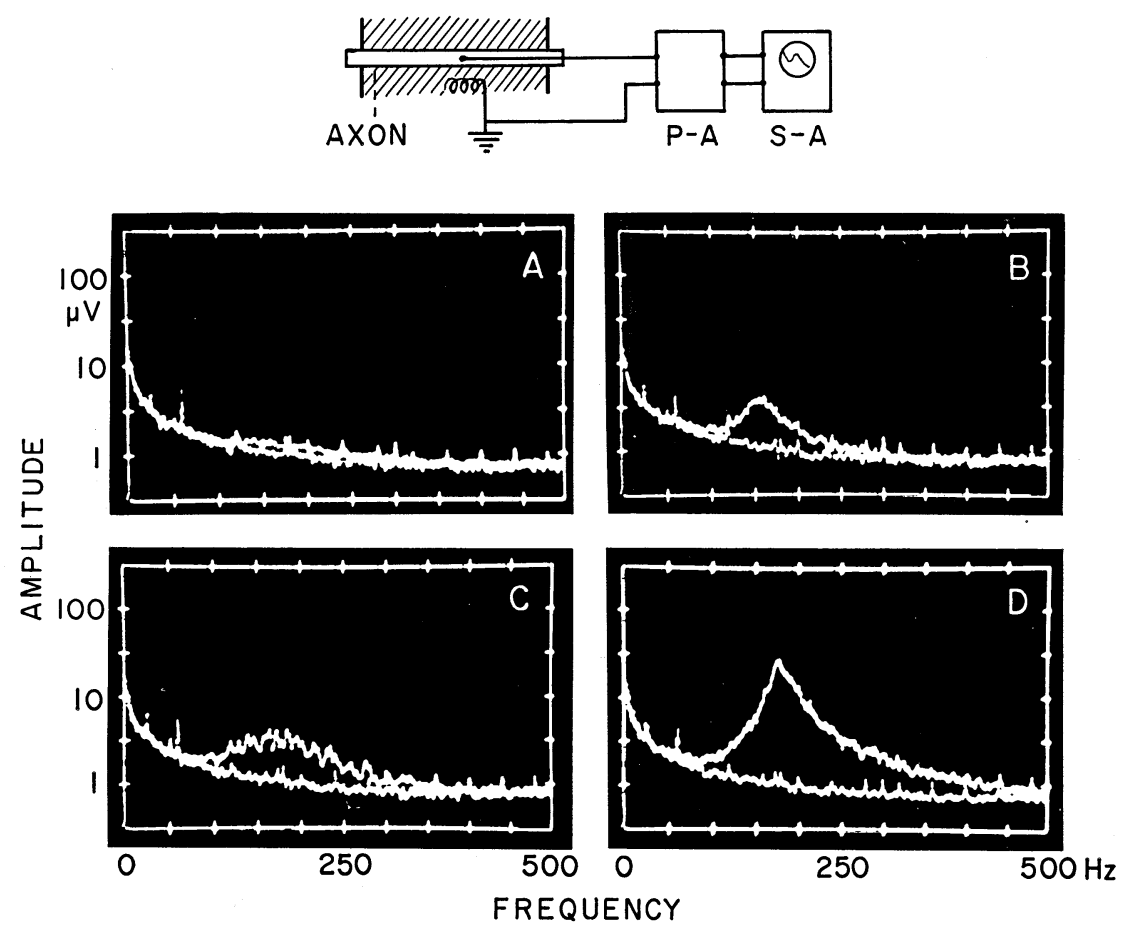

Fig. 1. Top: A schematic diagram illustrating the experimental setup used: AXON represent a giant nerve fiber of the squid; P-A, a preamplifier with a high input impedance: S-A, a spectrum analyzer. Bottom: Four examples of oscillatory miniature responses. In each record, a spectrum was superposed which was taken with the recording electrode withdrawn from the interior of the nerve fiber and kept in the external medium. Record A was taken from an axon immersed in artificial sea water; note the existence of a small separation between the two oscillograph traces at about $150 \mathrm{~Hz}$. Records B, C, and D were obtained from axons immersed for 10-20 min in a medium consisting of one part sea water and three parts isotonic $\mathrm{NaCl}$ solution.

As can be seen in the examples of the records furnished in Fig. 1, the peak frequency of the spectrum did not vary appreciably among the axons examined. In almost all of the axons examined, it was found to be in the range between 140 and $190 \mathrm{~Hz}$. The half-bandwidth of the spectrum was generally between 60 and $100 \mathrm{~Hz}$. These spectra indicate that the frequency of the responses developed at individual sites in the membrane varies considerably from site to site.

When giant axons were kept in a medium with a reduced divalent cation con- 
centration, a stage was ultimately reached at which the signal amplitude increased very rapidly. This rapid increase in the amplitude was accompanied by a marked decrease in the half-bandwidth of the spectrum (see Record D in Fig. 1). This change in the characteristics of the spectrum took place when the amplitude exceeded roughly $50 \mu \mathrm{V}$ and could be attributed to an increased synchronization of the oscillatory responses of individual excitable sites due to an enhanced electric interaction between the sites. When this stage was reached, full-sized action potentials were released within a short period of time. These properties of chemically induced subthreshold responses are well known (see ARVANITAKI, 1939) and were not examined in the present studies. However, it is important to note that the frequency of these relatively large subthreshold responses is very close to the peak-frequency of the miniature responses described above. Furthermore, the frequency of repetition of full-sized action potentials induced in these intact (i.e., internally unperfused) axon is not very different from the peak-frequency of the miniature responses (Matsumoto et al., in preparation).

\section{The effects of temperature changes}

Since both the frequency and amplitude of oscillatory miniature responses were found to change very slowly with time, the effects of quick changes in the temperature could be examined by circulating two salt solutions (of the same chemical composition) kept at different temperatures alternately. At the time when a giant axon immersed in a medium with a reduced divalent cation concentration was developing oscillatory responses of 5 to $10 \mu \mathrm{V}$ in amplitude at room temperature, a sudden cooling of the fiber always brought about a distinct increase in the response amplitude associated with a marked fall in the peak-frequency (see Fig. 2). The effect of cooling on the peak-frequency was perfectly reversible. However, the amplitude of the miniature responses remained at a level slightly larger than the initial value when the temperature was raised after a short period of cooling.

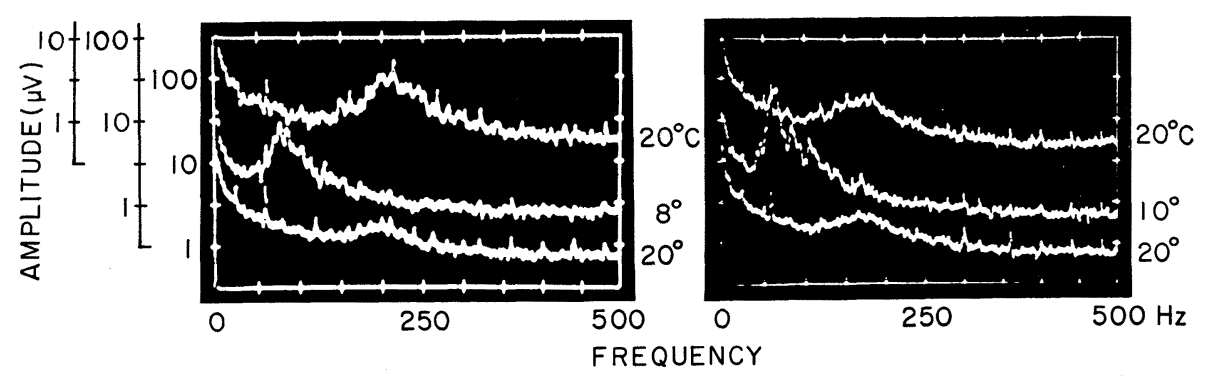

Fig. 2. Spectra showing the effect of temperature changes on the oscillatory miniature responses. The records on the left were taken from an axon immersed in a medium containing $7 \mathrm{~mm} \mathrm{CaCl}_{2}$ and $519 \mathrm{~mm} \mathrm{NaCl}$. For the righthand records, the external medium was a 1: 3 mixture of normal sea water and isotonic $\mathrm{NaCl}$ solution. The three vertical scales indicate how much the spectra in each record is displaced. 
The temperature quotient for the peak-frequency between 10 and $20^{\circ} \mathrm{C}$ was estimated to be roughly 2.3. However, because of the large variation among 10 different axons used for this purpose, the reliability of these measurements was quite limited.

\section{The effects of application of the salt of potassium ions}

The effects of various cations were tested by using squid axons immersed in a mixture of isotonic solutions of $\mathrm{NaCl}$ and $\mathrm{CaCl}_{2}$ (see METHODS). Oscillatory miniature responses were found to develop gradually when the Ca-ion concentration in the mixture was less than about $15 \mathrm{~mm}$. In axons developing miniature responses, replacement of a large portion of the Na-ions with an equivalent amount of potassium brought about a large change in the spectrum, usually a sudden increase in amplitude followed by a loss of the signal. These results were poorly reproducible.

Highly reproducible results were obtained when axons immersed in a medium with a relatively high $\mathrm{Ca}$-ion concentration were employed. In a medium of which the Ca-ion concentration was $20 \mathrm{~mm}$ or slightly more, giant axons did not develop oscillatory responses spontaneously, although they remained highly excitable. Under these conditions, addition of a small amount of $\mathrm{KCl}$ to the external medium immediately evoked miniature responses. The result was quite reproducible when the external $\mathrm{KCl}$ concentration was raised quickly from zero to about $25 \mathrm{~mm}$ with the $\mathrm{CaCl}_{2}$ concentration kept constant. The potential difference across the axonal membrane (measured with a glass-pipette electrode filled with isotonic $\mathrm{KCl}$ solution) was reduced by a few $\mathrm{mV}$ under these circumstances.

An interesting aspect of the effects of $\mathrm{KCl}$ introduced into the external medium is that the response amplitude falls gradually after reaching a maximum. In some

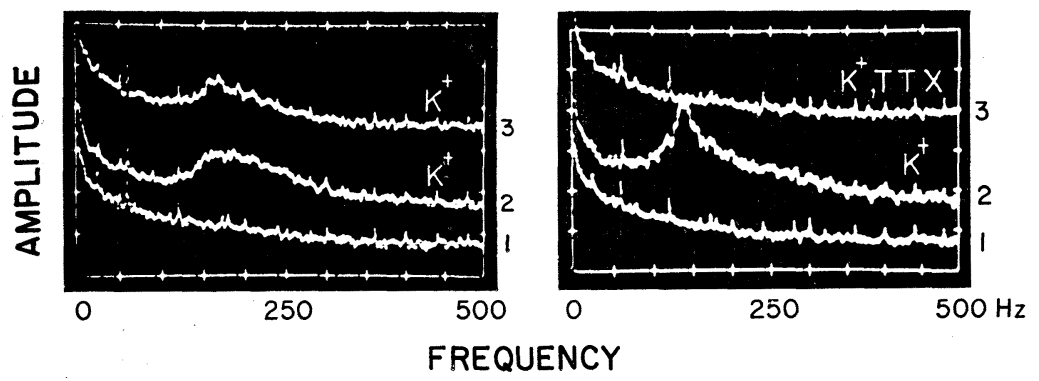

Fig. 3. Spectra of oscillatory miniature responses produced by external application of $\mathrm{KCl}$. The axons were immersed initially (spectrum 1) in a solution containing $20 \mathrm{~mm} \mathrm{CaCl}$ and $499 \mathrm{~mm} \mathrm{NaCl}$. Spectra 2 (right and left) were taken between 20 and $30 \mathrm{sec}$ after substitution of $24 \mathrm{~mm} \mathrm{KCl}$ for $\mathrm{NaCl}$ in the medium. The Ca-ion concentration was maintained at a constant level. Spectrum 3 on the left was taken about $90 \mathrm{sec}$ after spectrum 2 was taken. Spectrum 3 on the right was obtained about $1 \mathrm{~min}$ after addition of tetrodotoxin (TTX) to the medium $\left(8 \times 10^{-8} \mathrm{M}\right)$. 
instances, it took more than $20 \mathrm{sec}$ to reach a maximum; probably this delay is caused by the process of diffusion of $\mathrm{K}$-ions through the adherent tissue outside the axon. The responses were found to disappear almost completely within 4-5 min after $\mathrm{KCl}$ application. At this moment, the ability of the axon to develop full-sized action potentials was markedly depressed.

Another interesting feature of the action of $\mathrm{K}$-ions is the finding that the power spectrum of the responses induced was markedly asymmetric with respect to the vertical line drawn through the peak-frequency. An example of such highly asymmetric spectra is shown in Fig. 3, left; it is seen in the figure that signal extends widely toward the high frequency side of the peak. Similar asymmetric spectra were encountered when $\mathrm{Rb}$ - or Cs-salts were used instead of $\mathrm{K}$-salt (see later).

It is also interesting to note that the miniature responses evoked by external application of $\mathrm{KCl}$ could be suppressed by tetrodotoxin at a concentration sufficient to block full-sized action potentials (see trace 3 in Fig. 3, right). In this respect, the oscillatory responses evoked by $\mathrm{KCl}$ is not very different from those induced by other chemical means.

\section{Oscillatory responses evoked by electric currents}

The oscillatory responses described above were all induced chemically, namely, without using electric stimuli. The following observations show that application of electric current to the axonal membrane is a very effective means of evoking oscillatory miniature responses.

The experimental setup used in this series of experiments is illustrated schematically by the diagram in Fig. 4, top. Into a giant axon mounted horizontally in a Lucite chamber, a pair of glass cannulae filled with isotonic $\mathrm{KCl}$ solution $(\mathrm{C}$ in the figure) was introduced. One of the cannulae was slightly (approximately $4 \mathrm{~mm}$ ) shorter than the other. The electrode for recording oscillatory responses ( $\mathrm{R}$ in the figure) was then introduced into the axon at the other end in such a manner that the tip of the recording electrode was located midway between the two tips of the cannulae. The d.c. resistance of each of the cannulae (with a chloridized silver wire inside) was 3-4 megohms. By connecting a high resistance in series with each of these electrodes, the difference in the current intensity through the two electrodes was eliminated. This electrode arrangement was adopted in order to minimize the potential gradient around the tip of the recording electrode.

The external fluid medium used in these experiments contained approximately $20 \mathrm{mM} \mathrm{CaCl}_{2}$ and $500 \mathrm{~mm} \mathrm{NaCl}$. No clear sign of oscillatory responses were observed in axons immersed in this medium. A battery $(45 \mathrm{~V})$ and a potentiometer connected between the current electrodes and ground were used to apply currents to the axon. To eliminate the large transient variation in the membrane potential, the intensity of the applied current was increased gradually during a period of approximately $4 \mathrm{sec}$ (see METHODS).

It was shown by this method that passage of an outwardly directed current 

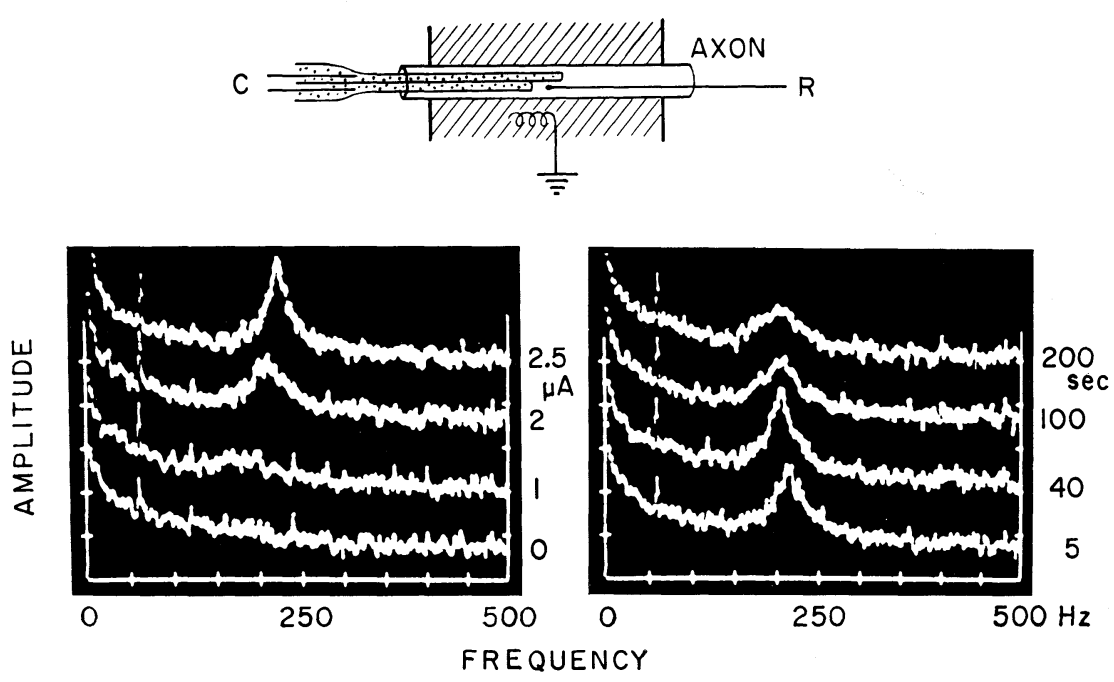

Fig. 4. Top: Schematic diagram showing the arrangement of the current $(C)$ and recording (R) electrodes used to study the effect of electric currents on the squid axon membrane. Bottom: Spectra of oscillatory miniature responses induced by outwardly directed membrane currents. Left: Records showing the dependence of the response amplitude on the current intensity; each photograph was taken approximately $10 \mathrm{sec}$ after the onset of the current. Right: Records showing a gradual change in the response amplitude after the onset of an outwardly directed current of $2 \mu \mathrm{A}$. These records were taken from an axon immersed in a medium containing $17 \mathrm{mM} \mathrm{CaCl}_{2}$ and $503 \mathrm{~mm} \mathrm{NaCl}(\mathrm{pH}$ 8.1) for approximately $20 \mathrm{~min}$.

through the axonal membrane produced oscillatory miniature responses which are very similar to those induced chemically. The power spectrum of these miniature responses appeared to be roughly Lorentzian with the peak-frequency located slightly below $200 \mathrm{~Hz}$ (see Fig. 4, left). As expected, the response amplitude increased sharply with the current intensity. As the response amplitude exceeded about $100 \mu \mathrm{V}$ peak-to-peak, there was a strong tendency toward initiating repetitive firing of action potentials preceded by marked sharpening of the spectrum. There was a slight rise in the peak-frequency as the current intensity was raised.

An important feature of the miniature responses induced by an outwardly directed membrane current is that the response amplitude decayed gradually with time when the current intensity was kept constant. Under the conditions of the present experiments, the time constant of this decay was of the order of $1 \mathrm{~min}$. This decay of the response amplitude was associated with a slight broadening of the spectrum. (Under the present experimental conditions, the rate of growth of the response could not be determined because of the gradual rise in the current intensity and the time required for averaging the signals.)

In axons that were not developing detectable miniature responses, application 
of an inwardly directed current to the membrane did not produce any visible effect. In axons generating distinct oscillatory responses, however, application of an inwardly directed current reduced or completely suppressed the responses. Under these circumstances, withdrawal of the applied current produced a transient increase in the response amplitude. When the applied inward current was strong enough, full-sized action potentials could be released immediately after the withdrawal of the applied current.

The apparent d.c. resistance of the axonal membrane under these conditions was estimated both by measuring the potential jump generated by a constant current pulse and by impedance measurements with low frequency a.c. The proportionality coefficient between the current intensity and the change in the membrane potential was, according to the present estimation, between 5 and 6 kilohms. From this it follows that the electric current used in the experiments described above produced sizable changes in the membrane potential. However, the change in the membrane potential per se may not be the cause of the production of the responses, since similar responses could be evoked in the absence of any membrane potential change.

\section{The effects of cesium, rubidium, lithium and procaine}

The effects of alkali metal ions (other than sodium and potassium) were examined by replacing $\mathrm{NaCl}$ in the surrounding medium with the equivalent amount of $\mathrm{CsCl}, \mathrm{RbCl}$ or $\mathrm{LiCl}$. During this procedure, the concentrations of other ions in the medium (divalent cations, anions, hydrogen ion and tris buffer) were kept unaltered.

An example of the results obtained with cesium is presented in Fig. 5, left. The axon under study was immersed in a mixture of isotonic solutions of $\mathrm{NaCl}$ and $\mathrm{CaCl}_{2}$ (see the figure legend). At the time when the axon was developing relatively small oscillatory responses, $10-20 \%$ of the total $\mathrm{Na}$-ion was replaced with Cs-ion. This replacement brought about a distinct increase in the response amplitude accompanied by broadening of the spectrum and lowering of the peakfrequency. In some instances, full-sized action potentials were released at the peaks of large subthreshold responses. As in the case of $\mathrm{KCl}$, there was a gradual decay of the responses during the course of 1-2 min after replacement of $\mathrm{CsCl}$ for $\mathrm{NaCl}$. The results obtained from 9 different axons were all quite consistent. In each of these axons, the effects mentioned above could be demonstrated for several times.

The effect of substitution of $\mathrm{Rb}$-ions for $\mathrm{Na}$-ions were examined by the same procedure using 4 different axons. The results obtained were very similar to those for $\mathrm{K}$-ions.

The results obtained with Li-ions were very different from those with other cations. It was found that substitution of $20-30 \%$ of the external $\mathrm{Na}$-ions with Li-ions almost completely suppresses the miniature responses generated in a me- 
dium with a reduced divalent cation concentration (see Fig. 5, middle). This suppression was observed in all (9) axons examined. When the Li-ions in the medium were replaced with Na-ions, the responses reappeared immediately. A similar suppressive action of Li-ions was observed previously by OzEKI et al. (1966).

The effect of procaine hydrochloride on oscillatory miniature responses was examined on 5 different axons. In the range of concentration sufficient to block production of action potentials, the miniature responses were suppressed completely. This effect was almost completely reversible (see Fig. 5, right).
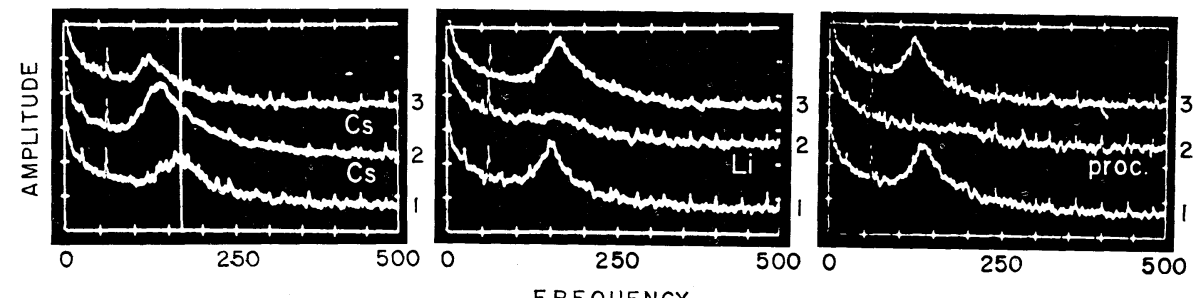

Fig. 5. Spectra showing the effects of $\mathrm{CsCl}, \mathrm{LiCl}$ and procaine hydrochloride on the oscillatory miniature responses. Left: The axon was kept in a medium containing $17 \mathrm{~mm}$ $\mathrm{CaCl}_{2}$ and $503 \mathrm{~mm} \mathrm{NaCl}$ for about $10 \mathrm{~min}$ when the spectrum marked "l" was taken; spectra 2 and 3 were obtained 1 and 2 min, respectively, after substitution of $\mathrm{NaCl}$ with $\mathrm{CsCl}(80 \mathrm{~mm})$. Middle: The axon was immersed in a medium containing $7 \mathrm{mM} \mathrm{CaCl} \mathrm{Can}_{2}$ and $517 \mathrm{NaCl}$ for $10 \mathrm{~min}$ when spectrum 1 was taken; spectrum 2 was taken approximately 1 min after replacement of $1 / 3$ of the external sodium ions with lithium ions; spectrum 3 was obtained following the substitution of $\mathrm{Li}$ with $\mathrm{Na}$. Right: The axon was immersed in a medium containing $7 \mathrm{mM} \mathrm{CaCl}_{2}$ and $517 \mathrm{mM} \mathrm{NaCl}$ (spectra 1 and 3); spectrum 2 was taken about $1 \mathrm{~min}$ after substitution of $20 \mathrm{~mm}$ procaine hydrochloride for the external $\mathrm{NaCl}$ (keeping the Ca-concentration constant).

\section{DISCUSSION}

The afore-mentioned signals recorded from squid giant axons by the use of a real-time spectrum analyzer are extremely small in amplitude. It seems worthwhile, therefore, to show that these signals are not mere electric artefacts. One of the problems encountered in the present studies was how to reduce the artefacts arising from the power supplies at frequencies of $60 \mathrm{~Hz}$ and its integral multiples. A purely sinusoidal potential variation generates a sharp vertical line in the spectrum. In fact, such sharp vertical lines are recognizable in all the records presented in this paper. However, the spectra of signals under study are much broader and can easily be distinguished from the artefacts arising from the power supplies. Furthermore, a comparison of the spectra obtained with the recording electrode in and outside the axon (see Fig. 1) serves to identify the Fourier components of the non-axonal origin. Finally, the effects of various pharmacological agents (tetrodotoxin, tetraethylammonium, procaine, etc.) preclude the possibility that 
the signals discussed in this paper are physiologically unimportant artefacts. There is little doubt that the small signals recorded from squid giant axons derive from repetitive activation of macromolecules at a limited number of excitable sites of the axonal membrane. The power spectra of the axonal signals indicate that the electric responses at individual excitable sites repeat at a more-or-less regular interval. It has been noted that the spectra of small axonal responses are usually very irregular (see $e . g .$, Fig. $1, \mathrm{C}$ ), although the overall power distribution is roughly Lorentzian. (An explanation of the Lorentz formula in physics may be found, for example, in an article by VAN VLECK and WEISSKOPF (1945) or in a book by FRAUENFELDER (1963).) This fact suggests that the number of membrane sites involved is very small and the frequency of repetition varies to some extent from site to site. The present technique does not permit direct analysis of the timecourse of the responses at individual sites because of the long time-constant of the axonal membrane at rest.

It is expected that the electric interaction between individual excitable sites is negligible when the density of the sites involved is extremely small. The results of measurements of the electric impedance of the axonal membrane under these conditions have been interpreted as indicating that each excitable site responds to applied a.c. independently of others (Matsumoto et al., in preparation). The response amplitude increases as the number of sites involved increases. When the amplitude exceeds about $100 \mu \mathrm{V}$, however, there are definite signs of electric interaction between neighboring sites; the decrease in the bandwidth and the increased lability of the response spectrum are interpreted on this basis. Such large responses arising from mutually interacting excitable sites may properly be called "oscillatory subthreshold responses" of ARVANITAKI (1939). In contrast, the term "miniature responses" may be used to describe very small responses (less than about $30 \mu \mathrm{V}$ ) which show no sign of mutual interaction between individual sites.

It is important to note that development of oscillatory miniature responses induced by a reduction in the external divalent cation concentration (see Fig. 1) is not associated with any detectable fall in the electric (d.c.) potential difference across the axonal membrane. In other words, these responses are not triggered by a rise in the intracellular potential. The experimental findings described in this paper can readily be interpreted on the basis of the macromolecular theory in which conformational changes of the membrane macromolecules are assumed to be induced by an exchange between divalent and univalent cations within the macromolecules (TASAKI, 1968). The negative fixed charges of the macromolecules are neutralized, in the resting state, predominantly by divalent cations (Ca-ions), and replacement of a certain fraction of these divalent cations with alkali metal ions brings about a sudden conformational change of the macromolecules. A jump in the e.m.f. at the sites is regarded as the physico-chemical consequence of such a conformation change. The experimental findings described under Results indicates ability of alkali metal ions to displace $\mathrm{Ca}$-ions decreases in the following order: 


\section{$\mathrm{K}, \mathrm{Rb}>\mathrm{Cs}>\mathrm{Na}>\mathrm{Li}$.}

The finding that outwardly directed currents through the membrane evoke oscillatory miniature responses offers a basis for bridging the macromolecular theory with the ionic (equivalent circuit) theory of HoDGKIN and HUXLEY (1952). Since the cation present in the axoplasm are predominantly K-ions, transport of these cations is expected to bring about a transition of the macromolecules from the resting to the excited state conformation. Note that K-ions have the strongest tendency to displace $\mathrm{Ca}$-ions. A gradual decrease in the response amplitude under a continuous action of K-ions (see Figs. 3 and 4) may be attributed to a large increase in the fraction of the sites in the excited state resulting from irregular (asynchronous) transitions. Note that the spectra shown in Figs. 3, left and 4, top, are very broad. The processes that determine the frequency of miniature responses are not altogether clear at present. An investigation into these processes is now in progress.

The experimental results described in this paper show that the use of chemical stimulants and a real-time spectrum analyzer is a new means of studying properties of the excitable sites in the axonal membrane. One might say that the results obtained have thrown some new light onto the old problems in neurophysiology, such as para-resonance (MONNIER, 1933) and accommodation (NERNST, 1908), as well as onto the new problems, such as membrane noises in the squid axon membrane (Fishman et al., 1975; ConTr et al., 1975). It seems possible that this experimental technique is applicable to studies of excitable systems other than squid giant axons.

I thank Dr. Harold Gainer of N. I. H. for his comments on the problems discussed in this paper. I also thank Mrs. I. Tasaki who photographed all the spectra presented in this paper.

\section{REFERENCES}

ARVAnitaki, A. (1939) Recherches sur la réponse oscillatoire locale de l'axone géant isolé de sepia. Arch. Int. Physiol., 49: 209-256.

Brink, F., Bronk, D. W., and Larrabee, M. C. (1946) Chemical excitation of nerve. Ann. N. Y. Acad. Sci., 47: 457-485.

Conti, F., DeFelice, L. J., and Wanke, E. (1975) Potassium and sodium ion current noise in the membrane of the squid giant axon. J. Physiol., 248: 45-82.

Fishman, H. M., Pousart, D. J. M., and Moore, L. E. (1975) Noise measurements in squid axon membrane. J. Membr. Biol., 24: 281-304.

Frauenfelder, H. (1963) The Mössbauer Effect. W. A. Benjamin, Inc., New York (see equation 102 in Chapter 5).

Hodgkin, A. L. and Huxley, A. F. (1952) A quantitative description of membrane current and its application to conduction and excitation in nerve. J. Physiol., 117: 500-544.

InOUe, I., KobATAKe, Y., and TASAKI, I. (1973) Excitability, instability and phase transition in squid axon membrane under internal perfusion with dilute salt solutions. Biochim. Biophys. Acta, 307: 471-477.

Monnier, A. M. (1933) Phénomènes de "para-resonance" et théorie chronologique du systeme 
nerveux. C. R. Soc. Biol., 114: 1295-1297.

Nernst, W. (1908) Zur Theorie des elektrischen Reizes. Pflügers Arch. Physiol., 122: 275-314.

OzeKI, M., FreEman, A. R., and Grundfest, H. (1966) The membrane components of crustacean neuromuscular systems. I. J. Gen. Physiol., 49: 1319-1334.

TASAKI, I. (1968) Nerve Excitation: A Macromolecular Approach. Charles C. Thomas, Springfield, Ill.

VAN VleCK, J. H. and WeIsSKopf, V. F. (1945) On the shape of collision-broadened lines. Rev. Modern Phys., 17: 227-236. 